\title{
BMJ Open Association between sleep duration and stroke prevalence in Korean adults: a cross-sectional study
}

\author{
Min-Young Kim, ${ }^{1}$ Seunghoon Lee, ${ }^{2}$ You Ho Myong, ${ }^{2}$ Yoon Jae Lee, ${ }^{1}$ \\ Me-Riong Kim, ${ }^{1}$ Joon-Shik Shin, ${ }^{3}$ Jinho Lee, ${ }^{3}$ In-Hyuk Ha ${ }^{1}$
}

To cite: Kim M-Y, Lee S, Myong $\mathrm{YH}$, et al. Association between sleep duration and stroke prevalence in Korean adults: a crosssectional study. BMJ Open 2018;8:e021491. doi:10.1136/ bmjopen-2018-021491

- Prepublication history for this paper is available online. To view these files, please visit the journal online (http://dx.doi. org/10.1136/bmjopen-2018021491).

Received 3 January 2018 Revised 28 March 2018 Accepted 11 May 2018

Check for updates

${ }^{1}$ Jaseng Spine and Joint Research Institute, Jaseng Medical Foundation, Seoul, Korea

${ }^{2}$ College of Medicine, Seoul National University, Seoul, Korea ${ }^{3}$ Jaseng Hospital of Korean Medicine, Seoul, Korea

Correspondence to

Dr In-Hyuk Ha;

hanihata@gmail.com

\section{ABSTRACT}

Objectives Although sleep, chronic disease and its related mortality are extensively studied areas, the association between stroke and sleep duration is relatively unknown. The aim of this study was to investigate the association between long and short sleep duration and stroke prevalence.

Design A cross-sectional survey study.

Setting and participants Adult surveyees (aged $\geq 19$ years) who answered items relating to sleep duration and stroke in the 2010-2014 Korean National Health and Nutrition Surveys $(n=17601)$.

Outcome measures Participants were divided into three groups by sleep duration (short: $\leq 6$ hours/day, normal:

7-8 hours/day and long: $\geq 9$ hours/day). Stroke prevalence in each sleep duration group was compared using logistic regression analysis, and sociodemographic characteristics, medical history, lifestyle habits and mental health factors were set as confounding variables.

Results On adjusting for sex and age, each sleep-duration group displayed significantly different health-related characteristics. The short sleep and long sleep duration groups indicated significantly higher psychological factors for stress perception, depressive symptoms and psychiatric counselling compared with the normal sleep duration group. On adjustment of various confounders, the long sleep duration group demonstrated significantly higher ORs for stroke compared with the normal sleep duration group (OR 1.96, $95 \% \mathrm{Cl} 1.06$ to 3.61). Also, when stratified by sex, men did not exhibit differences in stroke prevalence by sleep duration, but women showed higher stroke prevalence in the long sleep duration group compared with normal sleep duration (OR $2.94,95 \% \mathrm{Cl}$ 1.21 to 7.17$)$.

Conclusions Longer sleep duration was associated with higher stroke prevalence, and this trend was more pronounced in women.

\section{INTRODUCTION}

Sleep is the daily process of recovering from a day's accumulated physical and mental fatigue and takes up to one-third of our lives. ${ }^{1}$ Determinants of sleep quality include quantitative factors such as total sleep time, sleep-onset latency and sleep maintenance (pertaining to sleep disruptions such as arousals and

\section{Strengths and limitations of this study}

The present study analysis was based on the results of a nationwide survey conducted by trained professionals in a systematic manner in a nationally representative sample of Koreans.

- The association between sleep duration and risk of stroke was investigated, adjusting for various confounders.

- The inherent nature of cross-sectional design studies, however, precludes this study from conclusively determining any potential causal relationships.

- Patient-reported outcomes are not free from recall bias, and serious stroke cases or cases in the elderly may have been inadvertently overlooked.

awakenings), and quantitative factors such as depth of sleep and sleep satisfaction, and both aspects are closely related to health, emotional state, and quality of life and satisfaction with life. ${ }^{2}$ Ideal sleep should meet a quantity and quality of an average 7-9 hours / day of sound sleep. ${ }^{3}$

The average life expectancy of Koreans as of 2016 was 79.3 years for men, and 85.4 years for women, which is 1.4 years and 2.3 years longer than that of the average of Organisation for Economic Co-operation and Development countries, respectively, and a 2.9-year increase for men and women aged 60 years compared with 10 years previous. However, the average healthy life expectancy, which is the life expectancy excluding disease duration, is discrepant at 64.9 years, and the importance of shortening disease duration in the elderly and extending healthy life expectancy through prevention, treatment and management of adult diseases continues to rise. ${ }^{4}$ Cerebrovascular diseases are the third leading cause of death in Koreans, ${ }^{5} 6$ and increased age is the largest risk factor for stroke for both sexes. ${ }^{7}$

Along with physical changes, changes to sleep patterns are a part of the normal ageing 
process. According to earlier studies, short sleep duration is related to the onset of diabetes, obesity, hypertension, coronary disease and stroke, and this has been shown to lead to increased mortality. ${ }^{8-10}$ Further, the difference in mortality by sleep duration draws a U-shaped graph that grows higher on both ends compared with the recommended sleep duration of intermediate range. This implies that overly long sleep is also significantly associated with mortality. ${ }^{9}{ }^{10}$ In a 2014 study of sleep duration and history of stroke in US adults analysed by sex and age, short or long sleep duration was shown to significantly increase the onset of stroke compared with the group with normal sleep duration. ${ }^{8}$ However, the association between sleep duration and stroke prevalence may differ by cultural region or ethnicity.

The association between sleep duration and stroke prevalence considering for age, sex and mental health has not been reported in Koreans as of yet. The hypothesis of this study was that abnormal sleep duration (short or long sleep duration) would be associated with higher stroke prevalence compared with normal sleep duration in Koreans, similar to that seen in other studies. This study thus investigates the association between sleep duration and stroke prevalence adjusting for various confounding factors such as age, sex, lifestyle habits and medical history using Korea National Health and Nutrition Examination Survey (KNHANES) data.

\section{METHODS}

\section{Participants}

This study was conducted using raw data from KNHANES. KNHANES is a national survey conducted for surveillance of the health and nutritional status of Koreans in accordance with Article 16 of the National Health Promotion Act, and the statistics are government-designated statistics as defined in Article 17 of the Statistics Act (approval number 117002). The statistics are provided yearly for health-related policies by the Korean Ministry of Health and Welfare and the Korea Centers for Disease Control and Prevention (KCDC) in the Korea Health Statistics, the official report of KNHANES. KNHANES was first established in 1998, and is a nationally representative cross-sectional survey conducted every year by the KCDC, and as such assesses health and dietary nutritional profiles and health-related behaviours in about 10000 individual household members aged 1 year and over from 23 households extracted by probability sampling of 192 primary sampling units. The surveyees are divided by life cycle stage characteristics into infants and children (age 1-11 years), adolescents (12-18 years) and adults (19 years or older), and survey contents vary according to these age characteristics. KNHANES consists of three component surveys: the health interview survey, health examination and nutrition survey. Health interview surveys and health examinations were performed at mobile examination centres, and nutrition data were collected through visits to the selected households a week afterwards. Further details are available on the KNHANES website (https:// knhanes.cdc.go.kr/knhanes/eng/index.do).

In order to increase the number of participants, the researchers combined data from 2010 to 2014. The number of participants in the raw data of the fifth KNHANES conducted in 2010-2012 was 31 596, and that of the sixth KNHANES conducted in 2013-2014 was 19814. The number of participants, who responded to the survey in each KNHANES period, was 25533 and 15568 , respectively. The researchers collected data of the 17601 participants who answered the self-questionnaire items pertaining to stroke prevalence and sleep duration.

\section{Stroke}

Patients who had stroke were defined as individuals who answered 'yes' to the health questionnaire item on stroke asking 'have you been diagnosed with stroke' to which the participants were asked to answer either 'yes' or 'no'.

\section{Sleep duration}

Average daily sleeping hours were written in a blank space in two digits by the hour, from 01 to 24 . For this study, the participants were categorised into groups of 6 hours of sleep or less (short sleep duration), 7-8 hours of sleep (normal sleep duration) and 9 hours of sleep or more (long sleep duration) a day.

\section{Covariates}

Participants, who were identified as having a history of stroke in the survey, were assessed for various sociodemographic variables including sex, age, income and educational level to adjust for confounding factors that may potentially influence sleep duration and stroke. In addition, lifestyle factors such as body mass index (BMI), drinking and smoking habits, and intensity of daily activities were investigated. Concerning medical history, bed rest for the past month, and dyslipidaemia, diabetes and hypertension prevalence were surveyed. Lastly, usual stress perception, depressive symptoms, thoughts of suicide and psychiatric counselling experience were assessed for evaluation of mental health. Missing values in confounding factors were excluded in adjusting for confounding factors in statistical analysis.

\section{Sociodemographic variables}

Sex was dichotomised into male and female, and age was assessed as a numerical value. Income level was classified into quartiles (low, middle low, middle high and high) by the equalised average monthly household income (monthly household income/number of household members). Education was classified into elementary school graduation or lower, middle school graduation, high school graduation and college graduation or higher.

\section{Lifestyle variables}

BMI $\left(\mathrm{kg} / \mathrm{m}^{2}\right)$ was classified as $<18.5,<23,<25$ and $\geq 25$ as assessed through anthropometric measurements. Smoking status was classified as non-smoker if the individual had never smoked, past smoker if the individual 
had smoked in the past and current smoker if the individual smoked at present. Indirect smoking was defined as 'exposure to indirect indoor smoking at work or home for an hour or more a day'. Drinking habits were trichotomised into less than once a month, less than five times a month and five times or more a month. Lastly, daily activity was classified into the following four categories: sedentary state, light activity, moderate activity and heavy activity, according to the amount of physical activity including such activities as walking and weight lifting during the week immediately previous to the survey.

\section{Medical history variables}

Participants were queried on bed rest from illness for the past month, and current dyslipidaemia, diabetes and hypertension prevalence. Participants were asked to answer either 'yes' or 'no' to medical history items on specific medical conditions. The definition of diabetes included fasting blood sugar disorder in this study, and hypertension was defined as 'systolic blood pressure $\geq 140 \mathrm{~mm} \mathrm{Hg}$, diastolic blood pressure $\geq 90 \mathrm{~mm} \mathrm{Hg}$ or medication intake for hypertension'.

\section{Mental health variables}

Usual stress perception was considered to be high when the participant answered that he or she felt 'great stress' or 'much stress', and to be low when the participant replied that he or she felt 'little stress' or 'did not feel stress'. Experience of depressive symptoms for 2 weeks or longer, thoughts of suicide in the past year and psychiatric counselling in the past year were divaricate through 'yes' or 'no' answers.

\section{Data analysis}

KNHANES is a sample survey of the entire South Korean population that uses stratified cluster sampling and weighted samples. The sampling plan of this study accordingly followed a multistage complex sample probability design using stratified, clustered variables and weighting, and all analyses were performed using SAS V.9.3 (SAS Institute) statistics package with $\mathrm{p}<0.05$ set as statistically significant. Continuous variables were presented as mean and SD, and nominal variables as frequency and percentage (\%). Difference in participant characteristics by sleep duration was analysed using Rao-Scott $\chi^{2}$ test (age was assessed as a continuous variable and analysed using analysis of variance. Multinomial logistic regression analysis of the complex sample design adjusting for covariates was performed to assess the association between mental health factors and sleep duration, and ORs and 95\% CIs were calculated.

\section{Consent to participate}

All participants gave written informed consent before study participation, and participant information was not shared with interviewers prior to the interview.

\section{Patient and public involvement}

Patients and the public were not involved in development of the research question or outcome measures, study design, or recruitment to and conduct of this study. There are no plans for the study results to be disseminated directly to participants.

\section{RESULTS}

In the current analysis of 2010-2014 KNHANES data, a total of 17601 adult participants aged $\geq 19$ years were divided into the following three groups by sleep duration: $\mathrm{n}=7369(42 \%)$ sleeping 6 hours or less (short sleep duration), $\mathrm{n}=8918$ (51\%) sleeping 7-8hours (normal sleep duration) and $\mathrm{n}=1314(7 \%)$ sleeping 9 hours or more (long sleep duration). Stroke prevalence was 2\% ( $\mathrm{n}=360)$ in total participants who had answered the health survey questionnaire.

Participant characteristics by sleep duration are presented in table 1 . The mean age of participants in the short sleep duration group was $47.9 \pm 16.0$ years, and significantly higher than that of the normal sleep duration group. Lower income and educational levels were more prevalent in the long sleep duration group. Higher BMI values were more prevalent in the short sleep duration group, and lower BMI scores tended to present at a higher frequency in the groups of normal and long sleep duration. The group with 7-8 hours sleep duration showed higher frequency of $1 \leq<5$ sessions/month of alcohol use, and higher frequency of light and moderate intensity activities compared with the short and long sleep duration groups. Individuals regularly engaging in sedentary and high intensity activities displayed a higher prevalence of $\geq 9$ hours sleep. Participants who had required bed rest from illness in the past month were more prevalent in the short sleep group than in the normal sleep duration group, and showed a higher prevalence in the short and long sleep duration groups compared with healthy individuals. Participants with dyslipidaemia, diabetes and hypertension were more prevalent in the short sleep duration group compared with participants without these medical conditions, respectively (table 1 ).

Regarding mental health by sleep duration, both men and women in higher stress-level groups slept shorter hours, and most individuals in the group reported to be under less stress slept 7-8 hours. Among individuals with depressive symptoms, thoughts of suicide or psychiatric counselling, more were in short or long sleep duration groups compared with individuals without these respective conditions. However, men who had experienced depressive symptoms for $\geq 2$ consecutive weeks did not show differences between the $\leq 6$ hours sleep and normal sleep duration group and instead presented a high frequency in the $\geq 9$ hours long sleep duration group, which was different from the collective whole. Furthermore, the association between prevalence of women who received psychiatric counselling and sleep duration was non-significant, unlike the collective whole and in men 
Table 1 Demographic characteristics of Korean adults who participated in 2010-2014 KNHANES aged $\geq 19$ years $(n=17601)$

\begin{tabular}{|c|c|c|c|c|}
\hline \multirow[b]{3}{*}{ Variables } & \multicolumn{3}{|c|}{ Sleep duration } & \multirow[b]{3}{*}{$P$ values* } \\
\hline & $\begin{array}{l}\text { Short } \\
\text { ( } \leq 6 \text { hours) }\end{array}$ & $\begin{array}{l}\text { Normal } \\
\text { (7-8hours) }\end{array}$ & $\begin{array}{l}\text { Long } \\
\text { ( } \geq 9 \text { hours) }\end{array}$ & \\
\hline & 7369 & 8918 & 1314 & \\
\hline Age $($ mean $\pm S D)$ & $47.9 \pm 16.0$ & $44.1 \pm 15.0$ & $44.7 \pm 18.2$ & $<0.0001$ \\
\hline \multicolumn{5}{|l|}{ Sex } \\
\hline Male & $3101(41.56)$ & 3852 (51.62) & $509(6.82)$ & 0.0017 \\
\hline Female & $4268(42.09)$ & $5066(49.97)$ & $805(7.94)$ & \\
\hline \multicolumn{5}{|l|}{ Household income } \\
\hline Low & $1787(42.23)$ & $2050(48.44)$ & $395(9.33)$ & $<0.0001$ \\
\hline Middle low & 1778 (40.35) & $2265(51.41)$ & $363(8.24)$ & \\
\hline Middle high & $1837(41.62)$ & $2276(51.56)$ & $301(6.82)$ & \\
\hline High & $1886(43.20)$ & $2239(51.28)$ & 241 (5.52) & \\
\hline \multicolumn{5}{|l|}{ Education } \\
\hline$\leq$ Elementary school & $2316(50.57)$ & 1815 (39.63) & $449(9.80)$ & $<0.0001$ \\
\hline Middle school & $833(43.07)$ & $953(49.28)$ & $148(7.65)$ & \\
\hline High school & $2209(38.48)$ & $3119(54.33)$ & $413(7.19)$ & \\
\hline$\geq$ College & $2006(37.58)$ & 3028 (56.72) & $304(5.70)$ & \\
\hline \multicolumn{5}{|c|}{ Body mass index $\left(\mathrm{kg} / \mathrm{m}^{2}\right)$} \\
\hline$<18.5$ & $271(34.57)$ & $422(53.82)$ & $91(11.61)$ & $<0.0001$ \\
\hline$<23$ & $2782(39.61)$ & 3675 (52.33) & $566(8.06)$ & \\
\hline$<25$ & $1777(43.33)$ & 2057 (50.16) & $267(6.51)$ & \\
\hline$\geq 25$ & 2507 (44.73) & 2718 (48.49) & $380(6.78)$ & \\
\hline \multicolumn{5}{|l|}{ Smoking status } \\
\hline Non-smoker & $3137(41.98)$ & $3746(50.13)$ & $589(7.89)$ & 0.0539 \\
\hline Indirect smoker & $1295(41.35)$ & 1641 (52.39) & $196(6.26)$ & \\
\hline Past smoker & $1370(42.55)$ & $1608(49.93)$ & $242(7.52)$ & \\
\hline Current smoker & $1484(41.57)$ & 1818 (50.92) & $268(7.51)$ & \\
\hline \multicolumn{5}{|l|}{ Drinking } \\
\hline$<$ Once/month & 4344 (42.74) & $5030(49.49)$ & $789(7.77)$ & 0.0008 \\
\hline$<$ Five times/month & $1435(38.69)$ & 2015 (54.33) & $259(6.98)$ & \\
\hline$\geq$ Five times/month & 1567 (42.96) & $1819(49.86)$ & $262(7.18)$ & \\
\hline \multicolumn{5}{|l|}{ Level of daily activity } \\
\hline Sedentary & $788(40.81)$ & $872(45.16)$ & $271(14.03)$ & $<0.0001$ \\
\hline Light activity & 2918 (40.42) & $3820(52.92)$ & $481(6.66)$ & \\
\hline Moderate activity & $3026(43.25)$ & $3546(50.68)$ & $425(6.07)$ & \\
\hline Heavy activity & $632(43.89)$ & $674(46.81)$ & $134(9.30)$ & \\
\hline \multicolumn{5}{|l|}{ Bed rest for past month } \\
\hline No & $6684(41.43)$ & $8283(51.34)$ & $1166(7.23)$ & $<0.0001$ \\
\hline Yes & $684(46.69)$ & $633(43.21)$ & $148(10.10)$ & \\
\hline \multicolumn{5}{|l|}{ Dyslipidaemia } \\
\hline No & $6697(41.21)$ & 8325 (51.23) & $1229(7.56)$ & 0.0002 \\
\hline Yes & 672 (49.78) & 593 (43.93) & 85 (6.29) & \\
\hline \multicolumn{5}{|l|}{ Diabetes } \\
\hline No & $4637(40.12)$ & $6086(52.66)$ & $835(7.22)$ & 0.0002 \\
\hline Yes & 2131 (44.46) & 2318 (48.36) & $344(7.18)$ & \\
\hline
\end{tabular}


Table 1 Continued

\begin{tabular}{|c|c|c|c|c|}
\hline \multirow[b]{3}{*}{ Variables } & \multicolumn{3}{|c|}{ Sleep duration } & \multirow[b]{3}{*}{ P values* } \\
\hline & $\begin{array}{l}\text { Short } \\
\text { ( } \leq 6 \text { hours) }\end{array}$ & $\begin{array}{l}\text { Normal } \\
\text { (7-8hours) }\end{array}$ & $\begin{array}{l}\text { Long } \\
\text { ( } \geq 9 \text { hours) }\end{array}$ & \\
\hline & 7369 & 8918 & 1314 & \\
\hline \multicolumn{5}{|c|}{ Hypertension } \\
\hline No & $4750(39.46)$ & 6409 (53.24) & 879 (7.30) & $<0.0001$ \\
\hline Yes & $2583(47.10)$ & $2470(45.04)$ & $431(7.86)$ & \\
\hline
\end{tabular}

${ }^{*} \mathrm{P}$ value from ANOVA for continuous variables; Rao-Scott $\chi^{2}$ test for categorical variables.

ANOVA, analysis of variance; KNHANES, Korea National Health and Nutrition Examination Survey.

(table 2). When all psychological factors were considered, no significant associations were observed between stroke prevalence and psychological factors (table 3).
Table 4 presents the crude and adjusted ORs for risk of stroke by sleep duration with the normal sleep duration group set as reference. The ORs were primarily adjusted

Table 2 Association between sleep duration and psychological factors

\begin{tabular}{|c|c|c|c|c|c|}
\hline \multirow[b]{2}{*}{ Variables } & & \multicolumn{3}{|l|}{ Sleep duration } & \multirow[b]{2}{*}{ P values* } \\
\hline & & 7369 & 8918 & 1314 & \\
\hline \multicolumn{6}{|l|}{ Total } \\
\hline \multirow[t]{2}{*}{ Stress perception } & High & $2235(48.10)$ & $2084(44.85)$ & $328(7.05)$ & $<0.0001$ \\
\hline & Low & $5133(39.64)$ & $6830(52.75)$ & $985(7.61)$ & \\
\hline Depressive symptoms & Yes & $1100(46.67)$ & $1034(43.87)$ & $223(9.46)$ & \\
\hline \multirow[t]{2}{*}{ Thoughts of suicide } & No & $6146(40.91)$ & $7793(51.87)$ & 1086 (7.22) & $<0.0001$ \\
\hline & Yes & $1223(47.57)$ & $1121(43.60)$ & $227(8.83)$ & \\
\hline Psychiatric counselling & No & $7179(41.75)$ & $8747(50.87)$ & $1270(7.38)$ & $<0.0001$ \\
\hline Stress perception & Low & 2268 (39.53) & 3059 (53.32) & $410(7.15)$ & \\
\hline \multirow[t]{2}{*}{ Depressive symptoms } & No & $2797(41.21)$ & $3548(52.27)$ & $443(6.52)$ & 0.0009 \\
\hline & Yes & $304(45.10)$ & $304(45.10)$ & $66(9.80)$ & \\
\hline \multirow[t]{2}{*}{ Thoughts of suicide } & No & 2746 (40.95) & $3519(52.48)$ & $441(6.57)$ & 0.0006 \\
\hline & Yes & 355 (46.96) & $333(44.05)$ & 68 (8.99) & \\
\hline \multirow[t]{2}{*}{ Psychiatric counselling } & No & $3050(41.46)$ & 3809 (51.78) & $497(6.76)$ & $<0.0001$ \\
\hline & Yes & $51(48.11)$ & $43(40.57)$ & $12(11.32)$ & \\
\hline \multicolumn{6}{|l|}{ Women } \\
\hline Stress perception & High & $1402(47.98)$ & $1291(44.18)$ & $229(7.84)$ & $<0.0001$ \\
\hline Psychiatric counselling & Yes & $139(46.80)$ & $126(42.42)$ & $32(10.78)$ & \\
\hline
\end{tabular}

${ }^{*} \mathrm{P}$ value from Rao-Scott $\chi^{2}$ test. 
Table 3 Prevalence of stroke and psychological factors

\begin{tabular}{lrrr}
\hline \multicolumn{4}{c}{ Psychological factors } \\
\cline { 2 - 4 } & No & Yes & P values $^{*}$ \\
\hline History of stroke & & \\
No & $10949(63.52)$ & $6287(36.48)$ & 0.2803 \\
Yes & $210(58.33)$ & $150(41.67)$ & \\
\hline
\end{tabular}

${ }^{*} \mathrm{P}$ value from Rao-Scott $\chi^{2}$ test.

for sociodemographic characteristic variables (PR1). Secondary adjustments additionally included lifestyle variables (PR2), tertiary adjustments added medical history variables to PR2 (PR3) and lastly, quaternary adjustments covered mental health variables in addition to PR3 to consider all four categories of confounding factors (PR4). In analysis of total participants, the long sleep duration group indicated significantly higher OR values for stroke prevalence compared with the normal sleep duration group on adjusting for sociodemographic characteristic, lifestyle and medical history factors (OR 1.96, 95\% CI 1.06 to 3.61). However, risk of stroke did not exhibit associations with sleep duration despite adjusting for various confounding variables in men. Meanwhile, the long sleep duration group maintained high OR values compared with the normal sleep duration group in all models from PR1 to PR4 in adjustments for confounding factors in women. Of these results, the PR2 model that was adjusted for sociodemographic characteristics and lifestyle factors and the PR4 model that was adjusted for all variables exhibited statistically significant ORs for risk of stroke (OR 2.94, 95\% CI 1.21 to 7.17 ; OR 2.30, 95\% CI 1.01 to 5.21 , respectively) (table 4).

\section{DISCUSSION}

These study results show that sleeping for 9 hours or longer was partially associated with higher stroke prevalence compared with normal sleeping duration of 7-8 hours on adjusting for confounding factors. Especially in stratified analyses of men and women, while associations between sleep duration and stroke prevalence were non-significant in men, long sleep duration and higher stroke prevalence exhibited associations in women following adjustment for covariates.

Previous studies have shown that decreased sleep duration and quality of sleep is related to mental and physiological problems, which may affect metabolism and endocrine and immune systems and consequently exert a negative influence on quality of life. ${ }^{11}$ Fang et alconducted a study which showed that $29 \%$ or more adults in the US sleep 6 hours or less and $9 \%$ sleep 9 hours or more, and that these abnormal sleep durations had a significant association with higher stroke prevalence. ${ }^{8}$ In the multivariate

Table 4 Association between sleep duration and stroke

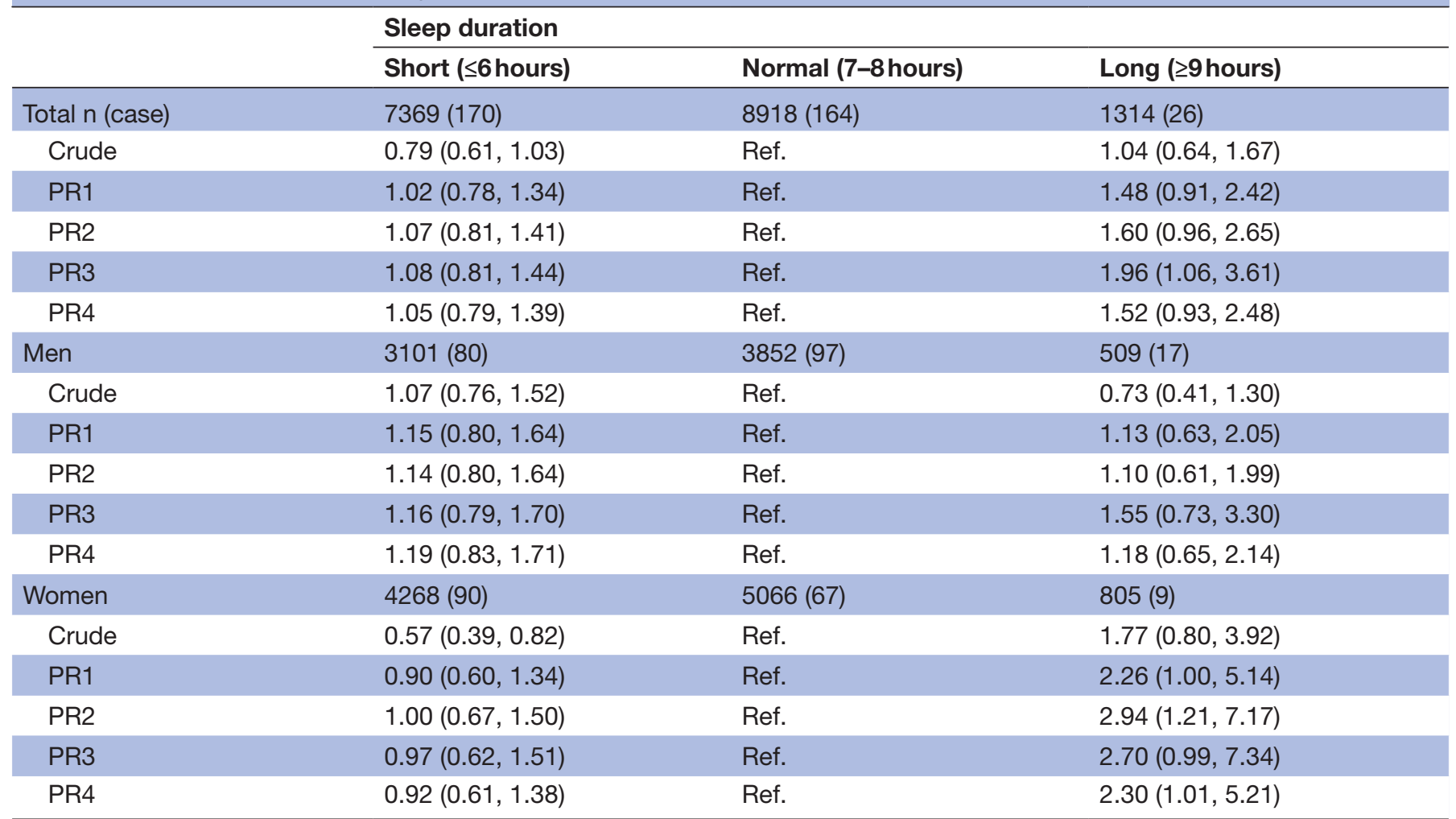

PR1: adjusted for age, household income and educational level.

PR2: adjusted for PR1 +smoking status, alcohol intake, daily activity for the past week and bed rest for the past month.

PR3: adjusted for PR2 +dyslipidaemia, diabetes, hypertension and body mass index.

PR4: adjusted for PR1 + psychological factors (stress, depressive symptoms, thoughts of suicide and psychological counselling). 
model adjusted for age and sex in this study, the $\leq 6$ hours sleep group and $\geq 9$ hours sleep group similarly indicated higher stroke prevalence compared with the 7-8hours sleep duration group. Moreover, with short or long sleep duration, prevalence of chronic diseases closely related to lifestyle such as depression, obesity, coronary heart disease and total cardiovascular disease, and all-cause mortality has also been shown to increase. This trend was consistently observed across several studies irrespective of nationality or ethnicity. ${ }^{9} 12$

While the association between sleep duration and onset of stroke has been demonstrated in several largescale population-based studies, the relationship in terms of sex and age has not yet been established due to the inconsistency of previous study results. A US study on sleep duration and cardiovascular disease which analysed 2005 National Health Interview Survey data in a nationally representative 1-year sample concluded that both $\leq 6$ hours and $\geq 8$ hours of sleep were associated with an increased likelihood of cardiovascular disease including stroke. ${ }^{13}$ A larger study conducted in a 6-year sample of US adults to the aim of assessing the association between sleep duration and history of stroke revealed that while long sleep duration was related to increased stroke prevalence in men and women aged $\geq 45$ in this population, shorter sleep duration was significantly related to increased stroke prevalence in women among younger adults aged 18-44. ${ }^{8}$ Ikehara et al observed 98634 Japanese participants over a course of 14.3 years during which 1964 participants died of stroke. Comparison of the group which slept 7 hours a day with 10 hours or more a day, adjusting for demographic characteristic, disease and lifestyle factors, indicated that long sleep duration increased the mortality of ischaemic stroke in both men and women. ${ }^{14}$ In a cohort study by Chen $e t a l$, 93175 American women aged between 50 and 79 years were followed up for 7.5 years, and it was discovered that stroke prevalence was significantly higher in the group of $\geq 8$ hours sleep duration compared with 7 hours in the 1166 cases of stroke onset. ${ }^{15}$ In addition, risk of stroke significantly increased along with snoring and excessive sleepiness in the group of $\geq 10$ hours sleep duration. However, although participants were perimenopausal due to the selective age range, risk of stroke was examined without considering for inherent sex and age characteristics including involutional depression, hormone replacement therapy and medical history, and the study failed to delineate possible mechanisms explaining the association between sleeping disorders and increased likelihood of stroke in women.

Sleep duration and cerebrovascular disease appear to hold associations through a number of different mechanisms. According to Spiegel et al, sleep deprivation causes functional metabolic disorder and leads to glucose shortage of the central nervous system which consequently raises blood pressure. ${ }^{16}$ Short sleep duration is also significantly related to elevated $\mathrm{C}$ reactive protein, which is an indicator of acute inflammation including that of the cardiovascular system. ${ }^{17}$ When this state is prolonged, various risk factors associated with cerebrovascular disease such as obesity, hypertension and cardiovascular disease may manifest. Qureshi et al reached the conclusion that individuals of $\geq 8$ hours sleep duration were approximately $50 \%$ more susceptible to stroke than those with normal sleep duration through 10 years of observation. This study also purported that sleep apnoea related to long sleep duration may decrease blood flow to the brain and increase intracranial pressure, thus heightening risk of stroke. ${ }^{18}$ The association between history of cerebrovascular disease and sleep duration appears to be bidirectional, with cerebrovascular disease affecting sleep duration and vice versa. Patients with stroke have been reported to suffer from sleep disorders caused by related symptoms and conditions such as fatigue, pain and depression. ${ }^{19-21}$ In a study conducted by Pasic et al, approximately 200 patients with stroke, $78 \%$ complained of sleeping disorders, and among them acute, haemorrhagic and right hemisphere stroke cases were found to be more prone to sleeping disorders. ${ }^{22}$ Prolonged central poststroke pain was also shown to impede quality of life, emotions, sleep and social functioning. ${ }^{23}$

There is a noted increase of research on the frequency of depressive disorders following the onset of stroke. Depression has been reported to be significantly related to both short and long sleep duration. ${ }^{24}$ Poststroke depression is the most common neuropsychiatric complication in stroke survivors, ${ }^{19}$ and reportedly attributes to low quality of life through impairment of physical function, restrictions in daily activity and altered sleep patterns including insomnia and increased napping. ${ }^{20} 25$

In this study, the difference in stroke prevalence associated with sleep duration was more conspicuous in women than in men. Although its cross-sectional design precludes the study from drawing any clear causal relationships, associations between sleep duration and stroke prevalence may be indirectly drawn based on differences in the female hormonal profile. Women may be more emotionally sensitive and aware of stress, and spend more time focusing on the cause of stress compared with men, ${ }^{26} 27$ and the frequency of sleep disorder symptom complaints and depression also tends to be higher. ${ }^{28-30}$ Moreover, de Zambotti et al reported that the night-time heart rate of perimenopausal women with insomnia is significantly higher in the follicular and luteal phases compared with ovulatory and menstrual phases, and confirmed that this may act as a risk factor for insomnia and cerebrovascular disorders. ${ }^{31}$ It may be inferred from these results that the hormonal change in middle-aged women affects the autonomic nervous system which controls and regulates homoeostasis, exposing women to higher risk of insomnia and cerebrovascular disorders.

The KNHANES data used in this study will inherently have been influenced by the sociocultural environment in Korea. Depression in Korean middle-aged women has been partially ascribed to termination of the traditional female role and gender identity, the economic 
independence of previously dependent children and change in appearance from hormonal depletion, ${ }^{32}$ and repeated episodes and chronicisation of these emotional changes may lead to disturbances in sleep patterns or physical symptoms such as heart palpitations, chest pain and headaches. ${ }^{30}$ However, the negative social perspective of psychiatric disorders and general lack of understanding of psychiatric counselling and medication is a major obstacle to patients seeking medical help in Korea ${ }^{33}$ It is conjectured that such social norms factored into the low use of psychiatric care reported in this survey.

Some strengths of this large-scale study include the fact that its study population is representative of the South Korean population, and that KNHANES adopted a yearround investigation system as of July 2007 and with it trained teams of professional investigators-including nurses, nutritionists and public health professionalsfor reliable survey investigation. The professional investigators were stationed at mobile examination centres following completion of 2-4 weeks of educational and supervised practical training sessions. As these professional investigators continued to receive regular retraining sessions to reinforce protocols and measurement techniques (7 sessions/year) and investigation performance was maintained through on-site quality control, although the survey items are self-reported, the dataset is construed to be of higher reliability than other comparable self-report surveys. In addition, various factors that can act as confounding variables for sleep duration and stroke (age, sex, income, educational level, drinking, cigarette smoking, BMI, daily activity, bed rest in the past month, hypertension, diabetes, dyslipidaemia, usual stress perception, depressive symptoms, thoughts of suicide and psychiatric counselling) were adjusted for. Nevertheless, there were various limitations to the study as well. Studies using KNHANES data are cross-sectional due to the inherent nature of the dataset, and as such related studies are able to investigate the potential association between two factors only within an arbitrary timeslot, and not longitudinally. Thus, inferring causality is not possible in this study design and only associations may be deduced. As mentioned above, it appears that stroke and sleep duration hold a mutually influential relationship through a variety of pathways and mechanisms. An added limitation is as the KNHANES health interview survey dataset is compiled through self-report questionnaires, only non-institutionalised patients with stroke who are capable of answering the questionnaire unassisted were included. Also, self-reporting in questionnaires renders them susceptible to recall bias due to subjectivity and poor memory, and possible misapprehension of symptoms mimicking stroke from such disorders as epilepsy, brain tumours or migraines. ${ }^{34}{ }^{35}$ Patients with stroke admitted to hospitals or sanatoriums, and deaths from stroke were also excluded from this study population. This study, therefore, holds the potential limitation of inadvertent data omission of serious stroke cases and stroke in elderly, institutionalised populations.
Further, while the study would have benefited from additional assessment of sleep apnoea and intake of such agents as sedatives and stimulants which may affect both sleep duration and stroke occurrence, KNHANES data did not include such items and thus limited more comprehensive assessment of the association between sleep duration and stroke prevalence.

\section{CONCLUSIONS}

Sleep duration exhibited significant differences by sociodemographic characteristics and medical history in Korean adults. Conditions such as hypertension, obesity, diabetes and dyslipidaemia were associated with short and/or long sleep duration, and sleep duration displayed distinct differences in presence of psychological factors in both men and women. Stroke prevalence was partially related to $\geq 9$ hours sleep duration when adjusted for confounding factors. While mental health factors and sleep duration revealed associations, mental health conditions were not directly associated with stroke. However, when variables including mental health were adjusted for, women with sleep duration of 9 hours or more were shown to be at higher risk of stroke. In conclusion, these study results partially validate the association between long sleep duration and higher stroke prevalence demonstrated in previous studies.

Although this study examined associations between stroke and sleep duration in Korean adults aged $\geq 19$ years adjusting for various sociodemographic covariates, future studies should consider focusing on high-risk populations and further evaluation of sleep patterns and lifestyle habits inclusive of sleep quality and related health conditions in addition to sleep quantity, using prospective study designs to reach more conclusive results. It is expected that furthering understanding of the mechanism underlying associations between stroke and sleep duration will contribute to advancement of stroke management and treatment.

Contributors M-YK, SHL, YHM and I-HH designed the study. M-YK, SHL, YHM, YJL, M-RK, J-SS and JL extracted and analysed the data. M-YK, SHL, YHM, YJL, M-RK and I-HH interpreted the results. M-YK and I-HH wrote the draft. SHL, YHM, YJL, M-RK, J-SS, JL and I-HH critically revised the manuscript. All authors read and approved the final version.

Funding This research received no specific grant from any funding agency in the public, commercial or not-for-profit sectors.

Competing interests None declared.

Patient consent Obtained.

Ethics approval The protocol was approved by the Institutional Review Board of Jaseng Hospital of Korean Medicine in Seoul, Korea (JASENG 2017-08-009).

Provenance and peer review Not commissioned; externally peer reviewed.

Data sharing statement Although there are no legal or ethical restrictions as to sharing the data publicly, the Korea National Health and Nutrition Examination Survey (KNHANES) data used in this study are third party data not owned by the authors. The data that support the findings of this study are available from the KNHANES website (https://knhanes.cdc.go.kr/knhanes/index.do) where the applicant may log onto and specify which annual report(s) are needed. The KNHANES annual reports, user manuals and instructions, and raw data are available on email request. Annual reports 2010-2014 were used in our study, and the 
authors confirm that others would be able to access these data in the same manner as they, as they did not have any special access privileges that others would not have.

Open access This is an open access article distributed in accordance with the Creative Commons Attribution Non Commercial (CC BY-NC 4.0) license, which permits others to distribute, remix, adapt, build upon this work non-commercially, and license their derivative works on different terms, provided the original work is properly cited and the use is non-commercial. See: http://creativecommons.org/ licenses/by-nc/4.0/

(C) Article author(s) (or their employer(s) unless otherwise stated in the text of the article) 2018. All rights reserved. No commercial use is permitted unless otherwise expressly granted.

\section{REFERENCES}

1. Yi H. Sleep quality and its associated factors in adults. Journal of Korean Public Health Nursing 2013;27:76-88.

2. Pilcher JJ, Ginter DR, Sadowsky B. Sleep quality versus sleep quantity: relationships between sleep and measures of health, well-being and sleepiness in college students. J Psychosom Res 1997;42:583-96.

3. Hirshkowitz M, Whiton K, Albert SM, et al. National Sleep Foundation's sleep time duration recommendations: methodology and results summary. Sleep Health 2015;1:40-3.

4. Statistics Korea. Life expectancy ( 0 years of age) and life expectancy for exclusion from illness. http://www.index.go.kr/potal/main/ EachDtIPageDetail.do?idx_cd=2758 (accessed Dec 26th 2017).

5. Korea S. Annual report on the cause of death statistics. Daejeon: Statistics Korea, 2016.

6. Kim C-G, Lee SH, Cha SK. Influencing factors on cardiocerebrovascular disease risk factors in young men: focusing on obesity indices. Journal of Korean Biological Nursing Science 2017;19:1-10.

7. Lee D-G. Risk factors of stroke. Korean Journal of Clinical Geriatrics 2002;3:5-16.

8. Fang J, Wheaton AG, Ayala C. Sleep duration and history of stroke among adults from the USA. J Sleep Res 2014;23:531-7.

9. Cappuccio FP, Cooper D, D'Elia L, et al. Sleep duration predicts cardiovascular outcomes: a systematic review and meta-analysis of prospective studies. Eur Heart J 2011;32:1484-92.

10. Liu TZ, Xu C, Rota M, et al. Sleep duration and risk of all-cause mortality: A flexible, non-linear, meta-regression of 40 prospective cohort studies. Sleep Med Rev 2017;32:28-36.

11. Birchler-Pedross A, Schröder CM, Münch M, et al. Subjective wellbeing is modulated by circadian phase, sleep pressure, age, and gender. J Biol Rhythms 2009;24:232-42.

12. Cappuccio FP, D'Elia L, Strazzullo P, et al. Sleep duration and all-cause mortality: a systematic review and meta-analysis of prospective studies. Sleep 2010;33:585-92.

13. Sabanayagam C, Shankar A. Sleep duration and cardiovascular disease: results from the National Health Interview Survey. Sleep 2010;33:1037-42.
14. Ikehara $\mathrm{S}$, Iso $\mathrm{H}$, Date $\mathrm{C}$, et al. Association of sleep duration with mortality from cardiovascular disease and other causes for Japanese men and women: the JACC study. Sleep 2009;32:295-301.

15. Chen JC, Brunner RL, Ren H, et al. Sleep duration and risk of ischemic stroke in postmenopausal women. Stroke 2008;39:3185-92.

16. Spiegel K, Leproult R, Van Cauter E. Impact of sleep debt on metabolic and endocrine function. Lancet 1999;354:1435-9.

17. Meier-Ewert HK, Ridker PM, Rifai N, et al. Effect of sleep loss on C-reactive protein, an inflammatory marker of cardiovascular risk. $J$ Am Coll Cardiol 2004;43:678-83.

18. Qureshi Al, Giles WH, Croft JB, et al. Habitual sleep patterns and risk for stroke and coronary heart disease: a 10-year follow-up from NHANES I. Neurology 1997;48:904-10.

19. Gaete JM, Bogousslavsky J. Post-stroke depression. Expert Rev Neurother 2008;8:75-92.

20. Kim M, Oh GJ, Lee YH. Association between stroke status and depression in a community setting: the 2014 Korea National Health and Nutrition Examination Survey. J Clin Neurol 2017;13:55-61.

21. Choi-Kwon S, Kim JS. Poststroke fatigue: an emerging, critical issue in stroke medicine. Int J Stroke 2011;6:328-36.

22. Pasic Z, Smajlovic D, Dostovic Z, et al. Incidence and Types of Sleep Disorders in patients with Stroke. Med Arch 2011;65:225.

23. Klit H, Finnerup NB, Jensen TS. Central post-stroke pain: clinical characteristics, pathophysiology, and management. Lancet Neurol 2009;8:857-68.

24. Gangwisch JE, Heymsfield SB, Boden-Albala B, et al. Sleep duration associated with mortality in elderly, but not middle-aged, adults in a large US sample. Sleep 2008;31:1087-96.

25. Suh M, Choi-Kwon S, Kim JS. Sleep disturbances after cerebral infarction: role of depression and fatigue. J Stroke Cerebrovasc Dis 2014;23:1949-55.

26. Feingold A. Gender differences in personality: a meta-analysis. Psychol Bull 1994;116:429-56.

27. Nolen-Hoeksema S. Sex differences in unipolar depression: evidence and theory. Psychol Bull 1987;101:259-82.

28. Krishnan V, Collop NA. Gender differences in sleep disorders. Curr Opin Pulm Med 2006;12:383-9.

29. Zhang B, Wing YK. Sex differences in insomnia: a meta-analysis. Sleep 2006;29:85-93.

30. Kessler RC. Epidemiology of women and depression. J Affect Disord 2003;74:5-13.

31. de Zambotti M, Trinder J, Colrain IM, et al. Menstrual cycle-related variation in autonomic nervous system functioning in women in the early menopausal transition with and without insomnia disorder. Psychoneuroendocrinology 2017;75:44-51.

32. Chang $\mathrm{H}$, Cha BK. [Influencing factors of climacteric women's depression]. Taehan Kanho Hakhoe Chi 2003;33:972-80.

33. Jung S-J, Lee S-S, Lee K-E, et al. Perception and attitude towards antidepressants in Koreans. Korean Journal of Clinical Pharmacy 2012.

34. Merino JG, Luby M, Benson RT, et al. Predictors of acute stroke mimics in 8187 patients referred to a stroke service. J Stroke Cerebrovasc Dis 2013;22:e397-e403.

35. Vilela P. Acute stroke differential diagnosis: stroke mimics. Eur J Radiol 2017;96:133-44. 